ARTICLE

\title{
Structural distortion and electron redistribution in dual-emitting gold nanoclusters
}

Qi Li ${ }^{1,2,6}$, Dongming Zhou ${ }^{3,6}$, Jinsong Chai ${ }^{1}$, Woong Young So (D) ${ }^{1}$, Tong Cai (i) ${ }^{4}$, Mingxing Li ${ }^{5}$, Linda A. Peteanu', Ou Chen (1) ${ }^{4}$, Mircea Cotlet $^{5}$, X. Wendy Gu (i) ${ }^{2 凶}$, Haiming Zhu (i) ${ }^{3 凶} \&$ Rongchao Jin (1) ${ }^{1 凶}$

Deciphering the complicated excited-state process is critical for the development of luminescent materials with controllable emissions in different applications. Here we report the emergence of a photo-induced structural distortion accompanied by an electron redistribution in a series of gold nanoclusters. Such unexpected slow process of excited-state transformation results in near-infrared dual emission with extended photoluminescent lifetime. We demonstrate that this dual emission exhibits highly sensitive and ratiometric response to solvent polarity, viscosity, temperature and pressure. Thus, a versatile luminescent nanosensor for multiple environmental parameters is developed based on this strategy. Furthermore, we fully unravel the atomic-scale structural origin of this unexpected excited-state transformation, and demonstrate control over the transition dynamics by tailoring the bitetrahedral core structures of gold nanoclusters. Overall, this work provides a substantial advance in the excited-state physical chemistry of luminescent nanoclusters and a general strategy for the rational design of next-generation nano-probes, sensors and switches.

\footnotetext{
${ }^{1}$ Department of Chemistry, Carnegie Mellon University, Pittsburgh, PA 15213, USA. ${ }^{2}$ Department of Mechanical Engineering, Stanford University, Stanford, CA 94305, USA. ${ }^{3}$ Centre for Chemistry of High-Performance and Novel Materials, Department of Chemistry, Zhejiang University, 310027 Hangzhou, Zhejiang, China. ${ }^{4}$ Department of Chemistry, Brown University, Providence, RI 02912, USA. ${ }^{5}$ Center for Functional Nanomaterials, Brookhaven National Laboratory, Upton, NY 11973, USA. ${ }^{6}$ These authors contributed equally: Qi Li, Dongming Zhou. ${ }^{{ }^{2}}$ email: xwgu@stanford.edu; hmzhu@zju.edu.cn; rongchao@andrew.cmu.edu
} 
$\mathrm{P}$ hotoexcitation can alter molecular structure and electron distribution ${ }^{1-17}$. This interesting phenomenon has endowed some organic molecules and metal complexes with two drastically different states, which are particularly appealing for applications in sensing, probes, switches, and actuators $^{1-17}$. However, this combined excited-state process has not yet been identified in nanomaterials, which is probably because of the increased structural rigidity and enhanced electron delocalization as materials transition from the molecular scale to the nanoscale. In the past decade, ultrasmall nanoparticles of $1-3 \mathrm{~nm}$ in diameter (often called nanoclusters) have attracted attention in the nanoscience community due to the atomic precision achieved in their synthesis and characterization and fascinating optical properties, such as the near-infrared (NIR) photoluminescence $(\mathrm{PL})^{18-29}$. We propose that nanoclusters can combine the merits of both larger nanoparticles (e.g., the longer fluorescence lifetime) and smaller molecules (e.g., the rich excited-state transformations), which can provide an exclusive opportunity for the development of new functional materials with synergetic properties.

Herein we directly identify a significant structural distortion accompanied by an electron redistribution in three photoexcited atomically precise nanoclusters $\left(\mathrm{Au}_{24}(\mathrm{~S}-\mathrm{TBBM})_{20}{ }^{30}, \mathrm{Au}_{14} \mathrm{Cd}_{1}(\mathrm{~S}\right.$ $\mathrm{Adm})_{12}{ }^{31}$, and $\mathrm{Au}_{24}(\mathrm{~S}-\mathrm{PET})_{20}{ }^{32}$, where $\mathrm{S}-\mathrm{TBBM}=4$-tertbutylphenylmethancan, S-Adm $=1$-adamantanethiol, and $\mathrm{S}$-PET $=$ 2-phenylethanethiol) using a combination of different spectroscopic techniques. The photo-induced changes in the structure and electron distribution in these nanoclusters lead to two significantly different excited states, thus dual emission is observed. The dual emission in these $\mathrm{Au}$ nanoclusters shows significant sensitivity to solvent polarity, viscosity, temperature, and pressure. Such dual emission with high sensitivity to multiple environmental parameters, together with the NIR II emission and the long PL lifetimes, are features that indicate that these $\mathrm{Au}$ nanoclusters are promising as next-generation probes and sensors. Furthermore, as the atomic structures of $\mathrm{Au}$ nanoclusters have been totally solved by the single-crystal X-ray diffraction $(\mathrm{XRD})^{18,30,31}$, the atomic-scale structural origin of such excitedstate transformation (structural distortion with electron redistribution) can now be fully unraveled. We find that the bitetrahedral core in Au nanoclusters is the fundamental structural origin of the excited-state structural distortion with electron redistribution, and the flexibility of the individual tetrahedra govern the excited-state transformation dynamics.

\section{Results}

Dual emission and excited-state dynamics of $\mathbf{A u}_{24}$. Figure 1 displays the atomic structure, steady-state optical spectra and PL dynamics of $\mathrm{Au}_{24}(\mathrm{~S}-\mathrm{TBBM})_{20}\left(\mathrm{Au}_{24}\right.$ for short hereafter.) Optical data were measured in dichloromethane (DCM). The atomic structure of $\mathrm{Au}_{24}$ (Fig. 1a) possesses a bi-tetrahedral $\mathrm{Au}_{8}$ core, in which the two tetrahedra are anti-prismatically joined together through two triangular faces (face to face). This bi-tetrahedral $\mathrm{Au}_{8}$ core is protected by four $\mathrm{Au}_{4} \mathrm{~S}_{5}$ surface motifs. The ultraviolet-visible (UV-Vis) absorption (black) and PL (gray) spectrum of $\mathrm{Au}_{24}$ are shown in Fig. 1b. In the absorption spectrum, a peak at $500 \mathrm{~nm}$ with a shoulder at $420 \mathrm{~nm}$ can be observed. Based on previous density functional theory (DFT) calculations, the $500 \mathrm{~nm}$ peak can mainly be attributed to the highest occupied molecular orbital-lowest unoccupied molecular orbital (HOMO-LUMO) transition, with a small contribution from the HOMO-2 to LUMO transition ${ }^{30}$. Both the HOMO and LUMO orbitals are distributed around the bi-tetrahedral $\mathrm{Au}_{8}$ core; thus the HOMO-LUMO transition occurs within the $\mathrm{Au}_{8}$ core ${ }^{30}$. The PL spectrum of $\mathrm{Au}_{24}$ (Fig. 1b, gray) in DCM shows dual emission, with one visible PL at $670 \mathrm{~nm}$ and one NIR PL at $1050 \mathrm{~nm}$. The overall quantum yield (QY) of $\mathrm{Au}_{24}$ is $2 \%$ using an integrating sphere. The PL excitation spectra for the two emission bands (Fig. 1c) were also measured. Both PL excitation spectra are similar as the UV-Vis absorption spectrum with the major peak at $\sim 500 \mathrm{~nm}$, indicating that both PL bands are excited by the $\mathrm{Au}_{8}$-core-based HOMO-LUMO transition.

The PL dynamics was studied by the time-correlated singlephoton counting (TCSPC) technique (Fig. 1d-f). Two lifetime components are required to fit the decays of PL I (Fig. 1d, detected from 550 to $750 \mathrm{~nm}$ ) and PL II (Fig. 1e, detected from 900 to $1000 \mathrm{~nm})$. The components of $1.6 \mathrm{~ns}(82 \%)$ and $77.1 \mathrm{~ns}$ (18\%) are required for PL I, and longer components of $66 \mathrm{~ns}$ (77\%) and $263 \mathrm{~ns}(23 \%)$ are required for PL II. Interestingly, when zooming into the early 2 -ns range (Fig. 1f), it can be observed that the fast decay of PL I corresponds to the rise of the PL II. This correspondence strongly suggests the direct electron transfer from PL I state to PL II state. This is the first time, to the best of our knowledge, that such a direct transfer event has been reported in metal nanoclusters.

To further unravel the excited-state dynamics of $\mathrm{Au}_{24}$ in DCM, a femtosecond transient absorption (fs-TA) study was conducted (Fig. 2 and Supplementary Fig. 1). The fs-TA spectra of $\mathrm{Au}_{24}$ at typical time delays within the first $1.4 \mathrm{~ns}$ are displayed in Supplementary Fig. 1. Rich electronic dynamics were observed within the 1.4-ns time window (the limit of our set-up). As shown in Fig. 2a, b, after pumping $\mathrm{Au}_{24}$ at $520 \mathrm{~nm}$, a ground-state bleaching (GSB) signal at $\sim 500 \mathrm{~nm}$ was immediately observed, which corresponds to the HOMO-LUMO transition. This GSB signal remains constant during the entire time window, indicating that no excited electrons relax back to the ground state in the 1.4ns period. On the other hand, three excited-state absorptions (ESAs) can be identified between 550 and $1300 \mathrm{~nm}$ (the red end of our window) and their time-dependent evolutions are significantly different. Starting from $\sim 100 \mathrm{fs}$ to $1 \mathrm{ps}$, one can observe a decay of the ESA centered at $\sim 600 \mathrm{~nm}$ and the rise of another ESA from 750 to $1200 \mathrm{~nm}$ (kinetic traces are displayed in Fig. 2b). More interestingly, starting from $\sim 10 \mathrm{ps,} \mathrm{one} \mathrm{can} \mathrm{observe}$ another significant electron transition process, which lasts until the end of our time-delay window (1.4 ns). It can be observed that there is a decrease of ESA from 750 to $1200 \mathrm{~nm}$, accompanied by the rise of a new ESA from 600 to $850 \mathrm{~nm}$. This nanosecond process during the TA measurement is consistent with the early time kinetics (within $1.5 \mathrm{~ns}$ ) observed in TCSPC measurements (Fig. 1f). The relaxation diagram of $\mathrm{Au}_{24}$ is displayed in Fig. 2c.

Ratiometric PL response to multiple environmental parameters. To obtain a deeper insight of the underlying mechanism of the dual emission and the state-to-state transition, the PL properties of $\mathrm{Au}_{24}$ were systematically studied in different environments (polarity, viscosity, solid state, and temperature). First, we describe the properties of $\mathrm{Au}_{24}$ in solvents of different polarity. The PL properties of $\mathrm{Au}_{24}$ in $\mathrm{DCM}$, toluene, and hexane are shown in Fig. 3a. The overall QY of $\mathrm{Au}_{24}$ increases from $2 \%$ in DCM to $10 \%$ in hexane. It can be observed that, from DCM to hexane (polarity decreases), the PL I significantly increases while the PL II slightly decreases. The UV-Vis absorption and PL excitation spectra of $\mathrm{Au}_{24}$ in hexane are similar to those in DCM, indicating no change of the ground state (Supplementary Fig. 2a, b). The lifetime of PL I shows a drastic increase to $>200 \mathrm{~ns}$ from DCM to hexane, and the fast sub-nanosecond decay that is observed in DCM disappears in hexane (Fig. 3b). In DCM, the lifetime of the PL I state is predominantly determined by this subnanosecond non-radiative decay, which arises from the state-tostate transition. Transferring $\mathrm{Au}_{24}$ from DCM to low-polarity 
a

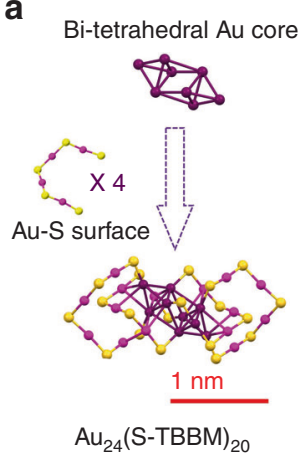

b

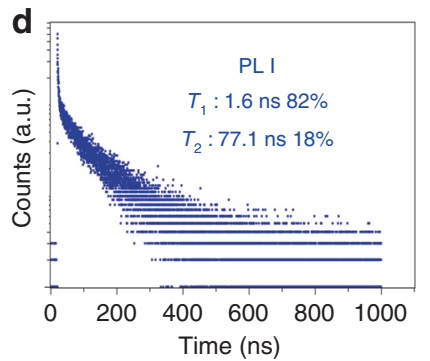

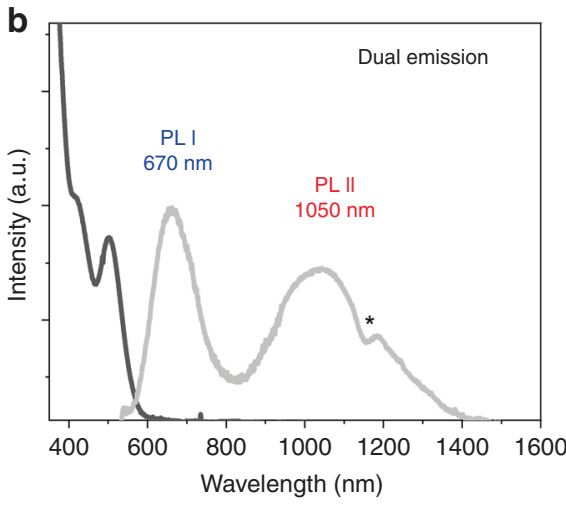
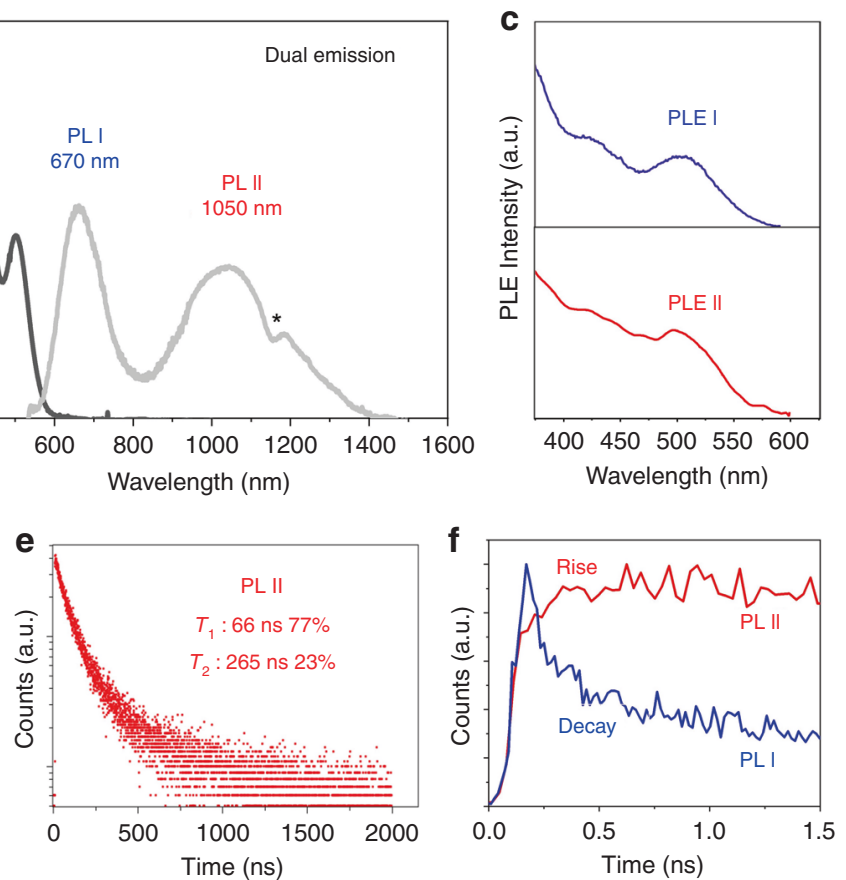

Fig. 1 Optical properties and PL dynamics of $\mathbf{A u}_{\mathbf{2 4}}$. a Anatomy of the atomic structure of $\mathrm{Au}_{24}$ determined by single-crystal $\mathrm{X}$-ray diffraction ${ }^{30}$. Purple $=$ core $\mathrm{Au}$; magenta = surface $\mathrm{Au}$; yellow $=\mathrm{S}$; Carbon tails are omitted for clarity. $\mathbf{b}$ UV-Vis absorption (black) and PL (gray) spectra (the asterisk denotes the spectrum "structuration", which is induced by the solvent). c PL excitation spectra for the two emissions, measured at $650 \mathrm{~nm}$ (blue) and $1050 \mathrm{~nm}$ (red), respectively. d, e Time-correlated single-photon counting (TCSPC) trajectories of the PL I (detected from 550 to $750 \mathrm{~nm}$ ) and PL II (detected from 900 to $1000 \mathrm{~nm}$ ). f Comparison of the TCSPC trajectories of PL I (blue) and PL II (red) in the early $1.5 \mathrm{~ns}$. PL photoluminescence, $\tau$ PL lifetime component.
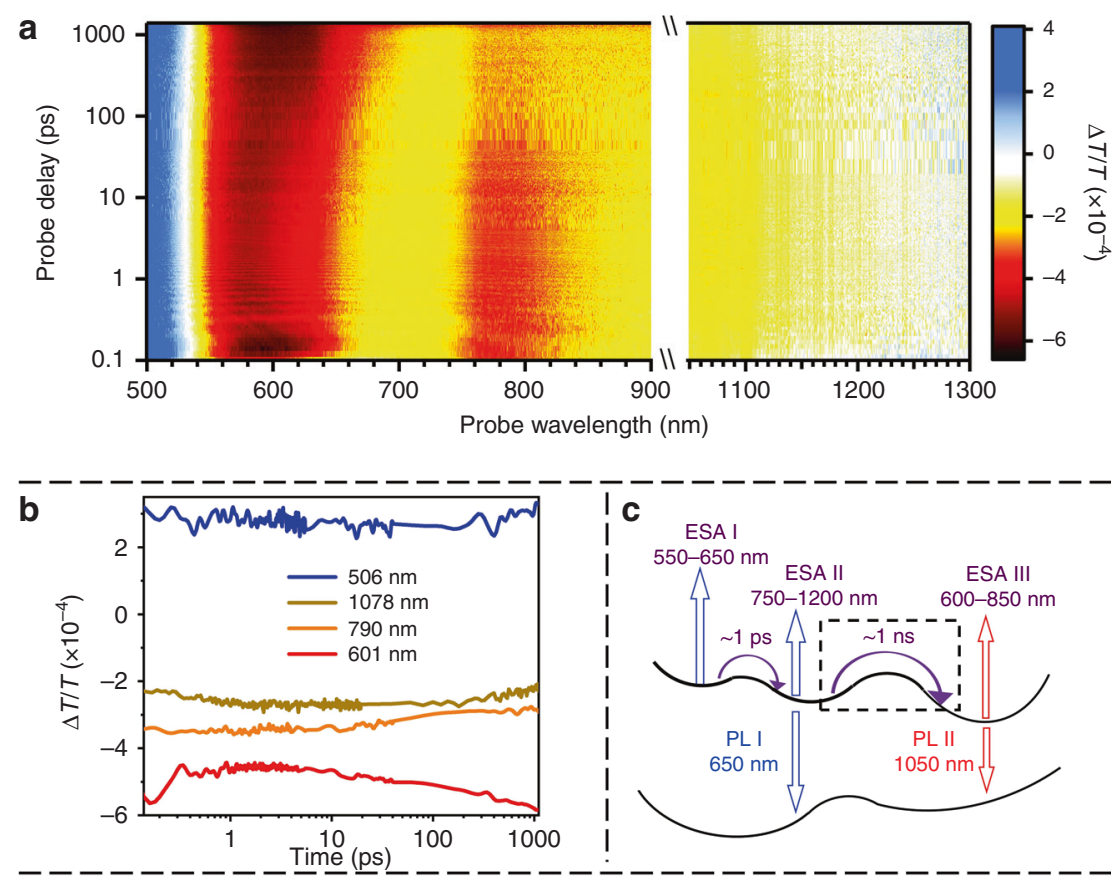

Fig. 2 Excited-state dynamics of $\mathbf{A u}_{\mathbf{2 4}}$. a Map of the $T A$ spectra of $A_{24}$ pumped at $520 \mathrm{~nm}$, which shows the $\Delta T / T$ at all time delays between 500 and $900 \mathrm{~nm}$ and between 1050 and $1300 \mathrm{~nm}$. b Selected kinetic traces at typical wavelengths. c Relaxation diagram for Au $\mathrm{Au}_{24}$. Arrows denote the transitions between different electronic states. PL photoluminescence, ESA excited state absorption.

solvents greatly slows down this process and thus significantly increases the overall PL efficiency of $\mathrm{Au}_{24}$.

We have also measured the PL spectra of $\mathrm{Au}_{24}$ in other solvents and in the solid state, as well as under liquid nitrogen. As shown in Fig. $3 c$ and Supplementary Figs. 3-5, significantly different PL spectra can be observed in these environments. From DCM to butanol, the QY of $\mathrm{Au}_{24}$ increases from $2 \%$ to $20 \%$, and no nanosecond decay can be observed in the TCSPC spectrum (Supplementary Fig. 3). QY up to $25 \%$ can be obtained in the solid state (crystal or film, Fig. 3c) and further increases to $~ 80 \%$ in liquid nitrogen (Supplementary Fig. 4). From DCM to liquid nitrogen, the significant increase ( 100 times $)$ and blue shift 

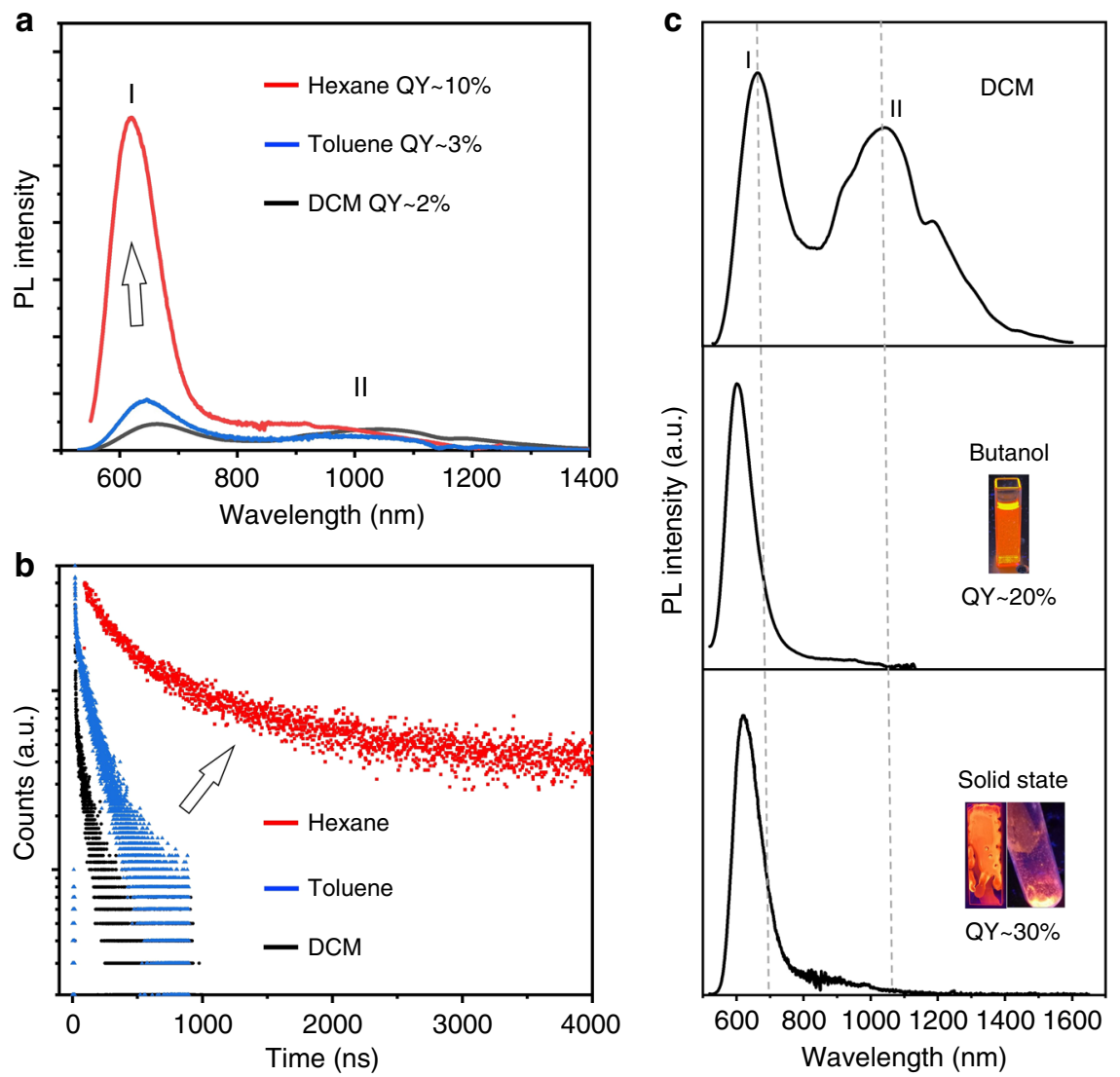

Fig. 3 Solvent polarity and viscosity dependence, as well as the solid-state PL of $\mathbf{A u}_{\mathbf{2 4}}$. Comparison of the PL (a) and TCSPC (b) of $A u_{24}$ in DCM (black), toluene (blue) and hexane (red). TCSPC trajectories were detected from 550 to $650 \mathrm{~nm}$. c Comparison of the PL of $\mathrm{Au}_{24}$ in DCM (top), butanol (middle), and solid state (crystal or film, bottom). From top to bottom, the overall QY of $\mathrm{Au}_{24}$ increases from $2 \%$ to $30 \%$, which is accompanied by an increase in the ratio of PL I/PL II and the blue shift of the PL I. Inset photographs: $\mathrm{Au}_{24}$ in butanol and solid-state film and single crystals under UV lamp. QY quantum yield.

(from 650 to $600 \mathrm{~nm}$ ) of PL I are observed, while the PL II almost remains unchanged and thus becomes negligible relative to PL I. This result suggests that the nanosecond transition from PL I state to PL II state is sensitive to the external environment.

Supplementary Table 1 summarizes the position of PL I in different environments. The first major conclusion that can be drawn from this data is that the PL of $\mathrm{Au}_{24}$ is dependent on the polarity of the solvent. The more polar the solvent, the more redshifted the PL (both I and II), and the stronger the PL II intensity. This polarity dependence suggests the charge-transfer nature in both PL states. The second conclusion is that the PL of $\mathrm{Au}_{24}$ is dependent on the solvent viscosity. In solvents with higher viscosity (i.e., butanol, octanol), significant enhancement of QY and increase of the PL I/PL II ratio were observed. And by adding more viscous solvent, the 1,2-dichlorobenzene into the DCM solution of $\mathrm{Au}_{24}$, it can be clearly observed that the nanosecond state transition gradually slows down (Supplementary Fig. 5). Such viscosity dependence suggests that the structure distortion happens between the two states ${ }^{14-17}$. This viscosity, together with the polarity dependence, are reminiscent of the twisted intramolecular charge-transfer model, which has been observed in 4-(dimethylamino)benzonitrile derivatives and other molecules $^{14-17}$.

To further probe the structure distortion under excitation, high-pressure PL measurements were conducted on the $\mathrm{Au}_{24}$. $\mathrm{Au}_{24}$ nanoclusters were loaded, along with toluene as a pressure medium, in a diamond anvil cell and compressed to $3.6 \mathrm{GPa}$ (Fig. 4a). As shown in Fig. 4b, the PL I significantly increases and blue-shifts under high pressure, and the PL II becomes negligible (note: the PL detector used in the high-pressure study is limited to $1000 \mathrm{~nm}$ ). This result can be explained if the photo-induced dynamic structure distortion is significantly hindered by the increase of the medium viscosity ${ }^{33}$ due to the high pressure, leading to the suppression of the excited-state transition. Our results also indicate that $\mathrm{Au}_{24}$ nanocluster can be applied as ratiometric pressure sensor.

Stark measurements were conducted to understand chargetransfer characteristic in $\mathrm{Au}_{24}$. Stark spectroscopy is a well-known method to reveal the charge-transfer mechanism ${ }^{34-36}$. Stark measurements provide information regarding intensity change (zeroth derivative component), change of polarizability (first derivative component), and change of dipole moment (second derivative component). For the electrofluorescence (EF) measurement (Fig. 5), excitation was performed at the wavelength where the electric field-induced change in absorption intensity was negligible (Supplementary Fig. 6a-c). From the EF measurement, the change of dipole moment (second) for the PL I of $\mathrm{Au}_{24}$ is found to be $1.07 \mathrm{D}$, indicating the partial charge-transfer occurrence at PL I, which is consistent with the solvent polarity dependence. As the HOMO and LUMO electrons are highly delocalized within the $\mathrm{Au}_{8}$ bi-tetrahedral core in $\mathrm{Au}_{24}{ }^{30}$, this partial charge transfer is better to be termed as electron redistribution, just as other conjugated molecules ${ }^{37}$. Unfortunately, our Stark spectra is limited up to $850 \mathrm{~nm}$, thus we are unable to verify the electron-redistribution character of PL II $(\sim 1050 \mathrm{~nm})$. 


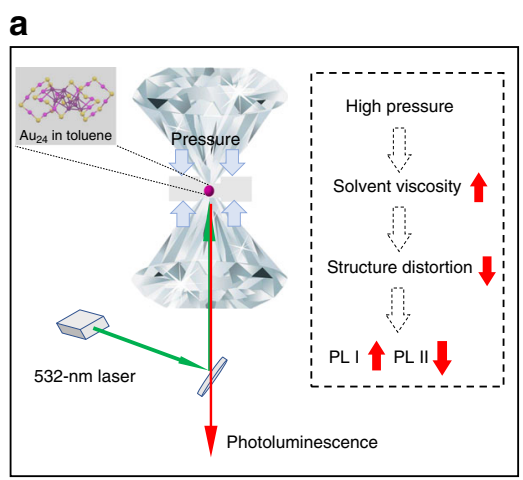

Diamond anvil cell high-pressure set-up b

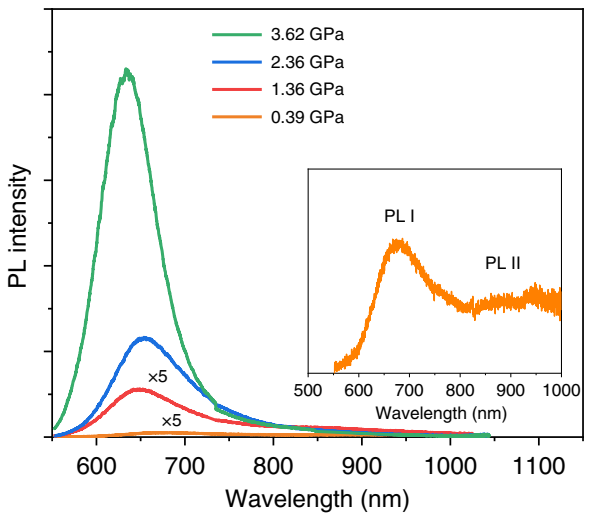

Fig. 4 Pressure-dependent PL study of $\mathbf{A u}_{\mathbf{2 4}}$. a The diamond anvil cell high-pressure set-up. $\mathrm{Au}_{24}$ in toluene is loaded inside the diamond anvil cell and compressed between the diamond anvils. Inset: mechanism of the pressure-induced change of the dual emission. $\mathbf{b}$ The pressure-dependent PL spectra of $\mathrm{Au}_{24}$. Inset: enlarged PL spectrum of Au24 at $0.39 \mathrm{GPa}$. PL photoluminescence.
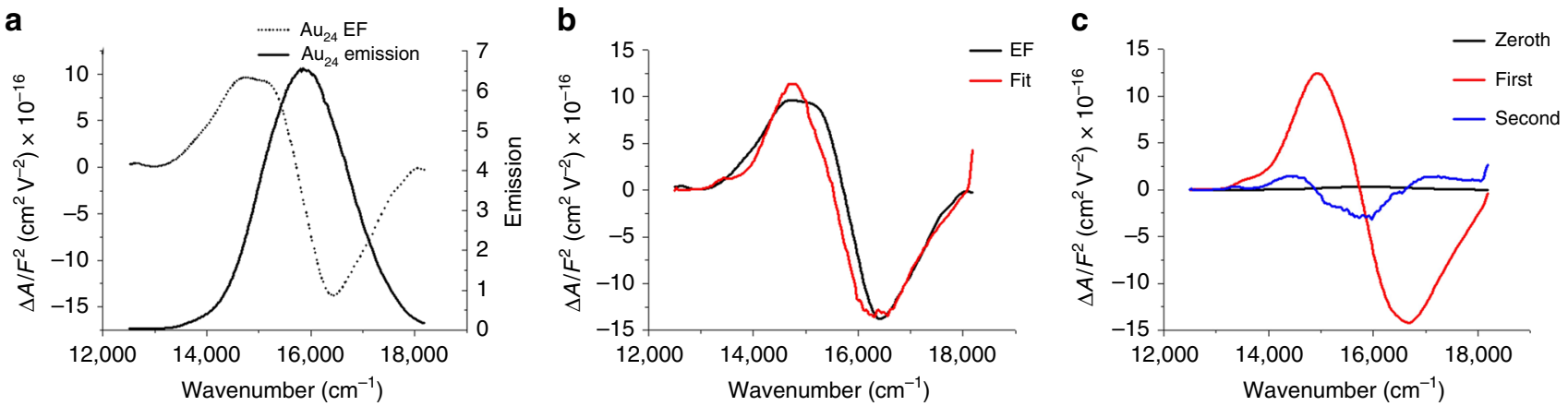

Fig. 5 Stark spectroscopic measurements on $\mathbf{A u}_{\mathbf{2 4}}$. a Emission (solid line) and electrofluorescence (EF; dashed). $\mathbf{b} E F$ (black) and fit (red, the details of the fitting are described in "Methods"). c EF fit broken into zeroth, first, and second derivatives.

Bi-tetrahedral series of Au nanoclusters with dual emission. In order to gain more structural insight of this nanosecond Au core distortion process, another bi-tetrahedral nanocluster, $\mathrm{Au}_{14} \mathrm{Cd}(\mathrm{S}$ $\mathrm{Adm})_{12}$, was also studied $\left(\mathrm{Au}_{14} \mathrm{Cd}\right.$ for short hereafter). Figure 6 displays the atomic structure, steady-state optical spectra, and excited-state dynamics of $\mathrm{Au}_{14} \mathrm{Cd}$ (optical data measured in $\mathrm{DCM})$. Anatomy of the atomic structure of $\mathrm{Au}_{14} \mathrm{Cd}$ is shown in Fig. 6a. The $\mathrm{Au}_{14} \mathrm{Cd}$ also possesses a bi-tetrahedral $\mathrm{Au}_{5} \mathrm{Cd}$ core, but each tetrahedron shares one edge with the other. This bitetrahedral $\mathrm{Au}_{5} \mathrm{Cd}$ core is further protected by two $\mathrm{Au}_{4} \mathrm{~S}_{5}$ motifs and one $\mathrm{AuS}_{2}$ motif. The UV-Vis absorption spectrum is shown in Fig. 6b (purple); a peak at $550 \mathrm{~nm}$ with a shoulder at $420 \mathrm{~nm}$ can be observed. Based on the previous DFT calculations ${ }^{31}$, this $550 \mathrm{~nm}$ peak can be mainly attributed to the HOMO-LUMO transition. Both the HOMO and LUMO are distributed around the bi-tetrahedral $\mathrm{Au}_{5} \mathrm{Cd}$ core and thus the HOMO-LUMO transition mainly occurs within the $\mathrm{Au}_{5} \mathrm{Cd}$ core ${ }^{31}$. The PL spectrum of $\mathrm{Au}_{14} \mathrm{Cd}$ (Fig. 6b, red) also shows dual emission, with one PL peak at $770 \mathrm{~nm}$ and the other at $800-900 \mathrm{~nm}$. The PL excitation spectrum of $\mathrm{Au}_{14} \mathrm{Cd}$ is similar as the UV-Vis absorption spectrum, and the main peak at $550 \mathrm{~nm}$ can be identified (Supplementary Fig. 7). As the two PL both originate from the corebased HOMO-LUMO transition, there is no obvious difference between the PL excitation spectra corresponding to PL at 770 and $900 \mathrm{~nm}$.

The PL dynamics was monitored at different wavelengths by the TCSPC technique. As shown in Fig. 6c, two lifetimes are needed to fit the decay at $850 \mathrm{~nm}$ : a short one of $334 \mathrm{ps}(93.8 \%)$ and a longer component of $86.6 \mathrm{~ns}(6.2 \%)$. When detected at shorter wavelengths (e.g., $680 \mathrm{~nm}$, see Supplementary Fig. 8), the PL decays faster with increasing amplitude of the short subnanosecond component. To further unravel the excited-state dynamics of $\mathrm{Au}_{14} \mathrm{Cd}$, fs-TA measurements were also conducted. As shown in Fig. 6d, e (TA spectra at typical time delays are shown in Supplementary Fig. 9a-c), after being pumped at $550 \mathrm{~nm}$, a GSB at $\sim 560 \mathrm{~nm}$ (corresponding to the HOMO-LUMO transition) can be immediately observed. Multiple ESAs can be identified and their time-dependent evolutions are significantly different. From the first $\sim 100 \mathrm{fs}$ to $\sim 1 \mathrm{ps}$, there is a decay of ESA at $\sim 730 \mathrm{~nm}$ accompanied by the rise of ESA centered at $\sim 670 \mathrm{~nm}$ (Fig. $6 \mathrm{~d}$, e and Supplementary Fig. 9a). The next process begins from 1 to $10 \mathrm{ps}$, it can be observed that a decrease of ESA centered at $\sim 630 \mathrm{~nm}$ is accompanied by a rise of the ESA at $700-950 \mathrm{~nm}$ (Fig. 6d, e and Supplementary Fig. 9b), which further decays to a new ESA from 650 to $850 \mathrm{~nm}$ from $10 \mathrm{ps}$ to $400 \mathrm{ps}$. Because of the significant overlap of the two ESAs, a clear decay can only be observed from 870 to $950 \mathrm{~nm}$. (Fig. 6d, e and Supplementary Fig. 9c) The abnormal increase of the GSB at $\sim 560 \mathrm{~nm}$ (the black curve in Fig. 6e) during this time period can be ascribed to a ESA decay located at $<600 \mathrm{~nm}$, which overlaps with GSB. Unfortunately, our current probe is limited to $>500 \mathrm{~nm}$, and no further information on this ESA can be obtained. The last process ( $\sim 150$ ps) is consistent with the $\sim 200-300$ ps component from the TCSPC measurements (Fig. 6c).

We studied the effects of different solvents on the optical properties of $\mathrm{Au}_{14} \mathrm{Cd}$. The PL spectrum of $\mathrm{Au}_{14} \mathrm{Cd}$ in hexane is shown in Supplementary Fig. 10 in which the second 800-900 nm peak becomes negligible. The transient absorption for $\mathrm{Au}_{14} \mathrm{Cd}$ in hexane was also measured (Supplementary Fig. 11a, b). In contrast to $\mathrm{Au}_{14} \mathrm{Cd}$ in $\mathrm{DCM}$, the electron relaxation to the 

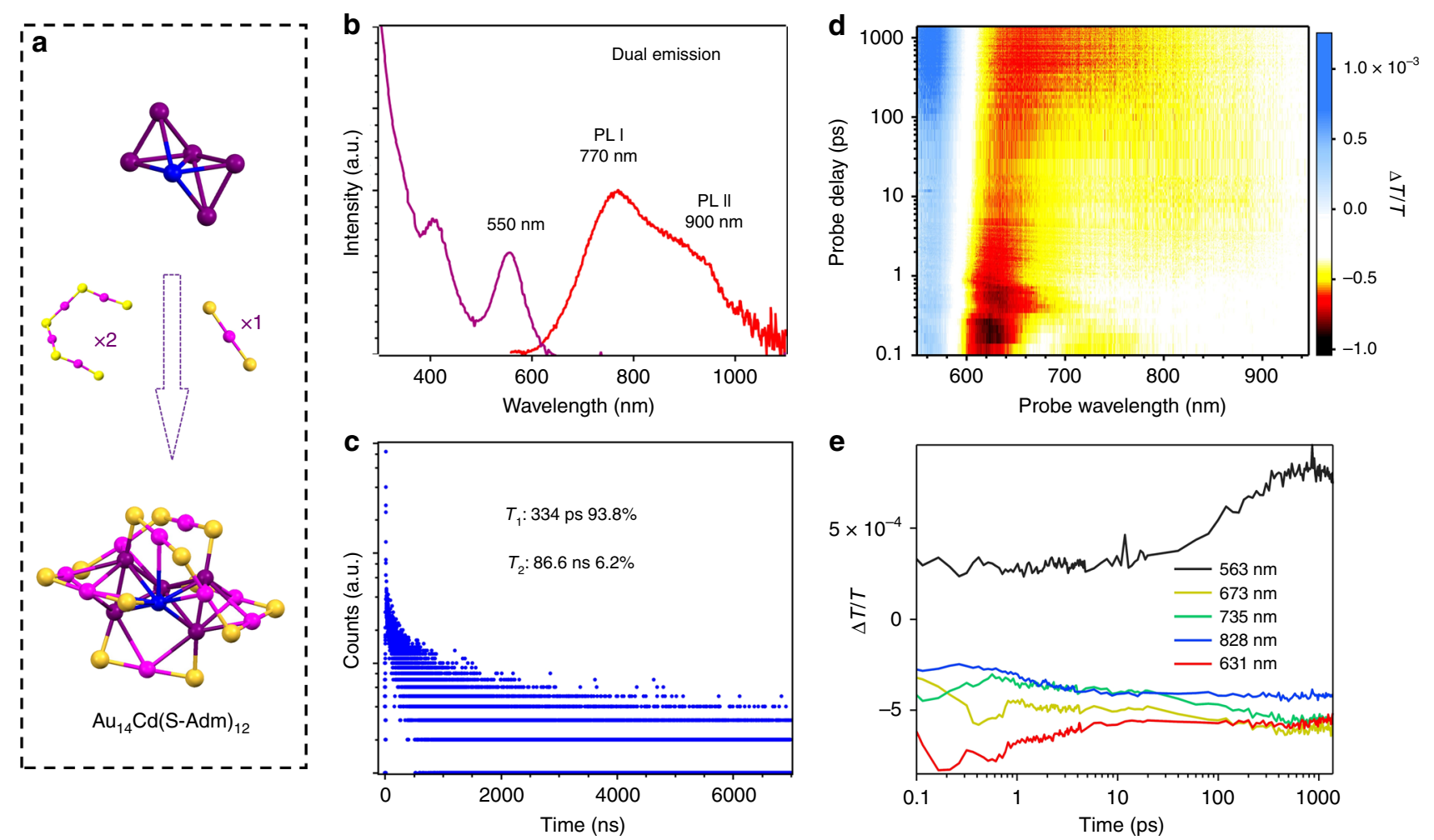

Fig. 6 Optical properties and excited-state dynamics of $\mathbf{A u}_{\mathbf{1 4}} \mathbf{C d}$. a Anatomy of the atomic structure of $\mathrm{Au}_{14} \mathrm{Cd}^{31}$. Purple $=$ core $A u$; magenta $=$ surface $A u_{;}$ blue $=\mathrm{Cd}$; yellow $=\mathrm{S}$; Carbon tails are omitted for clarity. (b) UV-Vis absorption (purple) and PL (red) spectra. PL photoluminescence. c TCSPC trajectory, which is detected at $850 \mathrm{~nm}$. $\tau$ PL lifetime component. d fs-TA spectra of $\mathrm{Au}_{14} \mathrm{Cd}$ pumped at $550 \mathrm{~nm}$, showing the $\Delta T / T$ at all time delays between 550 and $950 \mathrm{~nm}$. e Selected kinetic traces at typical wavelengths.

distorted electron-redistribution state has a slower transfer rate of $\sim 500$ ps (Supplementary Fig. 11b). In addition, a faster decay back to the ground state after $\sim 900$ ps was observed. The TA data are consistent with the PL and TCSPC results. For $\mathrm{Au}_{14} \mathrm{Cd}$ in hexane, owing to the slower state-transfer rate, a smaller fraction of electrons relaxed to the second distorted electron-redistribution state. This leads to the negligible PL II signal (Supplementary Fig. 10), and the faster decay of PL I (Supplementary Fig. 11b) to ground state is observed. Thus, when $\mathrm{Au}_{14} \mathrm{Cd}$ is dissolved in a lower dipole solvent, less electron redistribution to the distorted state occurs. This result suggests that similar electronredistribution mechanisms lead to dual emission in $\mathrm{Au}_{14} \mathrm{Cd}$ and $\mathrm{Au}_{24}$.

Thus similar relaxation diagrams for $\mathrm{Au}_{14} \mathrm{Cd}$ and $\mathrm{Au}_{24}$ are obtained from TA and TCSPC measurements. As shown in Fig. $7 \mathrm{a}$, for $\mathrm{Au}_{24}$, the excited electron first relaxes into the electron-redistribution state, which gives birth to the PL I at $\sim 650$ $\mathrm{nm}$. Through the next $\sim \mathrm{ns}$ process, the electron further relaxes into the distorted electron-redistribution state that results in the PL II at $\sim 1050 \mathrm{~nm}$. For the $\mathrm{Au}_{14} \mathrm{Cd}$ (Fig. $7 \mathrm{~b}$ ), the excited electron arrives at a similar electron-redistribution state, which gives birth to the PL I at $\sim 770 \mathrm{~nm}$. During the next $\sim 200 \mathrm{ps,} \mathrm{the} \mathrm{electron}$ further relaxes into the distorted electron-redistribution state, which is correlated to the PL II at $\sim 800-900 \mathrm{~nm}$. Compared with the $\mathrm{Au}_{24}$, the gap between the two states is smaller in $\mathrm{Au}_{14} \mathrm{Cd}$ $(0.24$ vs $0.68 \mathrm{eV})$, which is consistent with a faster transition rate ( $\sim 300$ ps vs $\sim 1.6 \mathrm{~ns})$. The smaller energy gap and faster transition between two states suggest that the structural difference of the two excited states in $\mathrm{Au}_{14} \mathrm{Cd}$ is much smaller than in $\mathrm{Au}_{24}$.

Other Au nanoclusters with bi-tetrahedral core structures have also been studied to further understand the mechanism of structural distortion and electron redistribution. Figure $7 \mathrm{c}$ displays another 24-gold-atom nanocluster protected by 20 phenylethalenethiol (S-PET) ligands (abbreviated as $\mathrm{Au}_{24}$ ' hereafter). The atomic structure of $\mathrm{Au}_{24}$ ' was determined by the combination of power XRD and simulations, which shows a different cross-joint bi-tetrahedral $\mathrm{Au}_{8}$ core protected by two $\mathrm{Au}_{3} \mathrm{~S}_{4}$ and $\mathrm{Au}_{5} \mathrm{~S}_{6}$ surface motifs ${ }^{32}$. It should be noted that this structure has also been verified in $\mathrm{Au}_{24}(\mathrm{SeR})_{20}$ nanocluster with 20 benzeneselenol as surface-protecting ligands ${ }^{38}$. As the selenium atom strongly interferes the excited-state processes through the heavy element effect, here we only discuss the optical properties of the PET-protected $\mathrm{Au}_{24}$ '. It can be observed that dual emission $(815 \mathrm{~nm}$ and $970 \mathrm{~nm})$ was also observed in the $\mathrm{Au}_{24}$ ' (Fig. 7c) and the state transition was determined to proceed in $\sim 700$ ps by the TCSPC and fs-TA measurement (Supplementary Fig. 12 and $13 \mathrm{a}-\mathrm{c}$ ).

We revisit the structural details of the bi-tetrahedral nanoclusters. In $\mathrm{Au}_{14} \mathrm{Cd}$, the two tetrahedra shares an edge (Fig. 7b), and this limits the free rotation of individual tetrahedra, thus the structural difference between the first electron-redistribution state and the second distorted electron-redistribution state is much smaller. In contrast, the connection and bonding between the two tetrahedra in $\mathrm{Au}_{24}$ are relatively weaker, and the two tetrahedra have more freedom to move under photoexcitation (Fig. 7a), which induces more significant differences between the two states. In addition, we found that, if there are several $(>2)$ tetrahedra existent in the core of the nanocluster (e.g., the monocuboctahedral series $^{39}$ and other larger face-centered cubic (fcc) $\mathrm{Au}$ nanoclusters ${ }^{40}$, Supplementary Fig. 14), the movement would be fully suppressed as every tetrahedron shares several edges and vertexes with other tetrahedrons (i.e., an interlocked kernel structure). Thus photo-induced structural distortion cannot be observed in the mono-cuboctahedral series ${ }^{39}$ and other fcc Au 
a
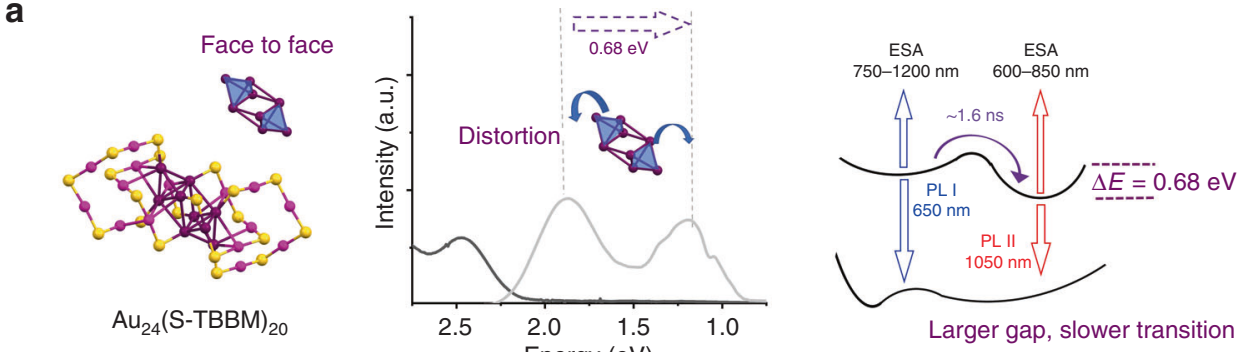

b--------------- - - Energy $(\mathrm{eV})---$
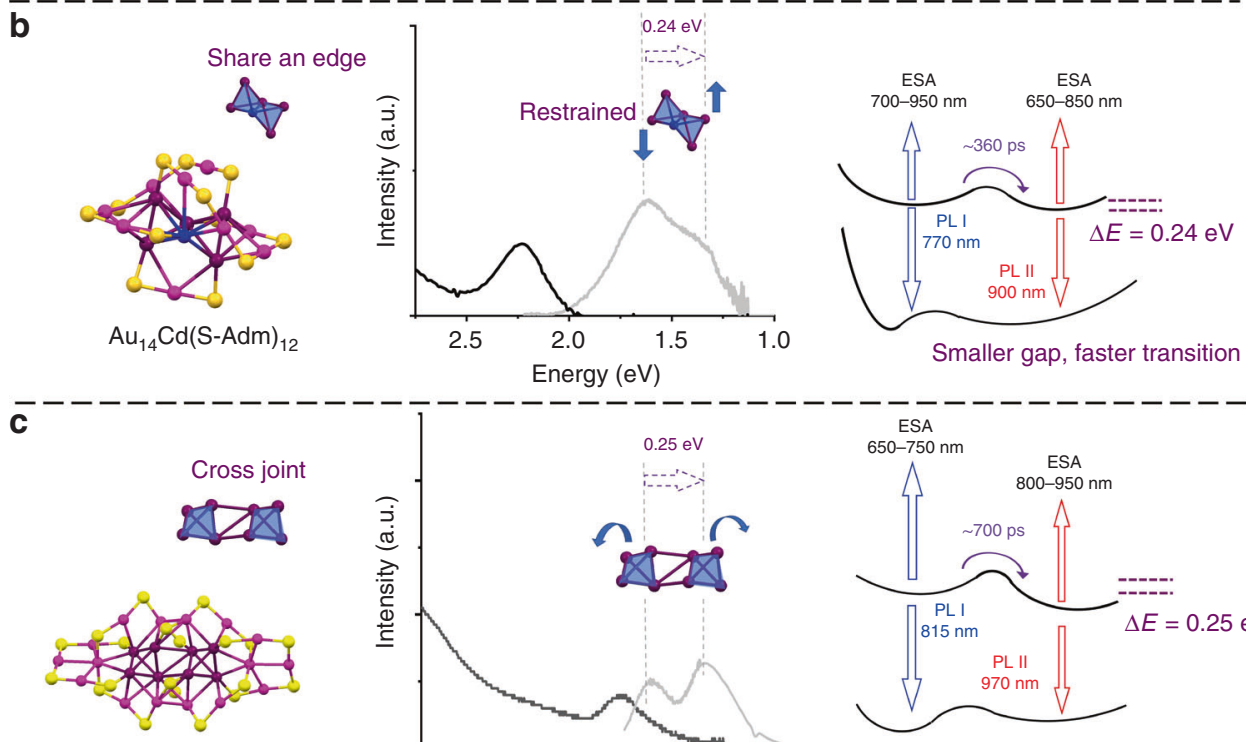

$\mathrm{Au}_{24}(\mathrm{~S}-\mathrm{PET})_{20}$
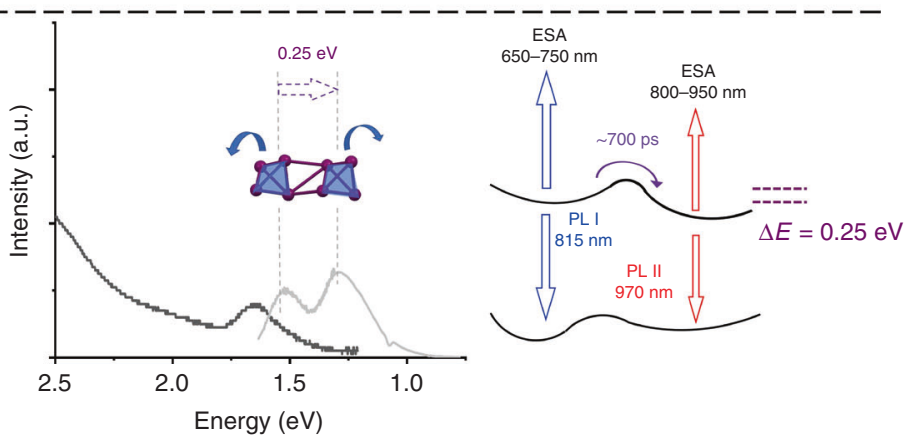

Fig. 7 Comparison of the three bi-tetrahedral series of $\mathbf{A u}$ nanoclusters. Atomic structure, optical properties, and relaxation diagram of $\mathbf{a} \mathrm{Au}_{24}, \mathbf{b} \mathrm{Au}_{14} \mathrm{Cd}$, and $\mathbf{c} \mathrm{Au}_{24}$ '. In each panel, the atomic structure of the nanocluster is shown on the left, purple = core $\mathrm{Au}$; magenta $=$ surface $\mathrm{Au}$; blue $=\mathrm{Cd}$; yellow $=\mathrm{S}$; the absorption (black) and PL (gray) spectra in the middle; and relaxation diagram on the right. Arrows denote the transitions between different electronic states. ESA excited state absorption.

nanoclusters ${ }^{40}$, even if tetrahedra exist in their structures. On the other hand, the core electrons in fcc nanoclusters with several $(>2)$ tetrahedra are more delocalized than in the bi-tetrahedral series. This explains that the charge-transfer characteristic (electron redistribution) can not be identified in fcc nanoclusters by the Stark spectroscopy ${ }^{39}$. The dependence of core structure indicates that the electron redistribution mainly take place within the metal $(0)$ core in bi-tetrahedral nanoclusters, which is different from the metal-ligand charge-transfer mechanism in traditional $\mathrm{Au}(\mathrm{I})$ and $\mathrm{Ag}(\mathrm{I})$ complex ${ }^{41,42}$. It should be noted that, owing to the ultrasmall size, the core atoms in Au nanoclusters are exposed to the outer environment in some directions (Supplementary Fig. 15), thus they can also interact with the solvents. It also should be noted that the photo-induced structure distortion can be treated as excited-state structural isomerization, thus one would question if there are already isomers in the samples. This possibility has been excluded by using high-purity samples that are re-dissolved from their single crystals, and these single crystals are examined by single-crystal XRD to confirm that no isomers exist inside.

\section{Discussion}

In summary, structural distortion and electron redistribution is unambiguously identified in photo-excited metal nanoclusters, and the atomic-scale structural origin of such excited-state transformation has been fully unraveled. This unexpected excited-state process in metal nanoclusters induces controllable dual emission, which exhibit highly sensitive and ratiometric responses to multiple external parameters, which makes these nanoclusters quite promising as next-generation probes and sensors. Overall, this work not only provides a simple but versatile strategy to achieve the self-calibrated luminescence responses to multiple environmental parameters from nanoclusters but also presents a paradigm in how to combine the merits of different scales of substances for the design of new materials with synergetic properties. We envision that the strategy and conception developed in this work will not only boost the research of both metal and other semiconductor nanoclusters but also advance the rational design of next-generation luminescent nanosensors, probes, and switches.

\section{Methods}

Sample preparation. The synthesis of $\mathrm{Au}_{24}, \mathrm{Au}_{14} \mathrm{Cd}$, and $\mathrm{Au}_{24}$ ' follow the published methods ${ }^{30-32}$.

Optical measurements. The UV-Vis absorption spectra were recorded using a Hewlett Packard 8543 diode array spectrophotometer. The PL spectra were recorded using a FS-5 fluorimeter from Edinburgh Instrument connected with two detectors from 200 to $870 \mathrm{~nm}$ and from 850 to $1600 \mathrm{~nm}$ and a Horiba Nanolog Hybrid Fluorimeter connected with an Ocean Optics 65000FL spectrograph/ charge-coupled device (CCD) $(400-1100 \mathrm{~nm})$, as well as a QM 40 spectrophotometer with a InGaAs $(500-1700 \mathrm{~nm})$ detector. The nanocluster solution is kept at $\sim 0.1$ optical density at the excitation wavelengths $\left(500 \mathrm{~nm}\right.$ for $\mathrm{Au}_{24}, 550 \mathrm{~nm}$ for $\mathrm{Au}_{14} \mathrm{Cd}$, and $750 \mathrm{~nm}$ for $\mathrm{Au}_{24}$ ') when measuring the PL spectra. The PL 
lifetimes were measured by a TCSPC technique with a femtosecond laser $(515 \mathrm{~nm})$ as the excitation source. TCSPC trajectories were fitted with bi-exponential functions. Temperature-dependent PL measurements were carried out on a Fluorolog-3 spectrofluorometer (Horiba Jobin Yvon) coupled with an Optistat DN cryostat (Oxford Instruments), an ITC temperature controller, and a pressure gauge. This homo-assembled system allowed us to conduct the temperature-dependent PL experiments from 298 to $80 \mathrm{~K}$. The Au nanoclusters were dissolved in 2methyltetrohydrofuran for temperature-dependent PL measurements.

Ultrafast spectroscopy. For fs-TA spectroscopy, the fundamental output from Yb:KGW laser (1030 nm, $220 \mathrm{fs}$ Gaussian fit, $100 \mathrm{kHz}$, Light Conversion Ltd) was separated into two light beams. One was introduced to NOPA (ORPHEUS-N, Light Conversion Ltd) to produce a certain wavelength for the pump beam (here we use $520 \mathrm{~nm}$ for the $\mathrm{Au}_{24}, 550 \mathrm{~nm}$ for $\mathrm{Au}_{14} \mathrm{Cd}$, and $750 \mathrm{~nm}$ for the $\mathrm{Au}_{24}{ }^{\prime}$ ), the other was focused onto a YAG plate to generate white light continuum as the probe beam. A 1030-nm laser was used to generate visible and NIR probe light, therefore the scattering region between 900 and $1050 \mathrm{~nm}$ in the data map is removed. The experiment set-up for the probes at $500-900 \mathrm{~nm}$ and $1050-1300 \mathrm{~nm}$ were different and used different pump power and pump laser beam size. Therefore, corrections are made to the pump fluences in the reported data. The pump and probe overlapped on the sample at a small angle $<10^{\circ}$. The transmitted probe light from sample was collected by a linear CCD array. DCM or hexane solutions of nanoclusters in $1 \mathrm{~mm}$ path length cuvettes were excited by the pump. Transient differential transmission signals were obtained by the equation shown below:

$$
\frac{\Delta T}{T}=\frac{T(\text { pump-on })-T \text { (pump-off })}{T(\text { pump-off })}
$$

Stark spectroscopy and data analysis. Electroabsorption (EA) and EF measurements were taken using a home-built Stark spectrometer ${ }^{43,44}$. White light from a 150 $\mathrm{W}$ Xe lamp (Oriel) is focused into the entrance slit of an Acton monochromator, which is equipped with a grating blazed at $500 \mathrm{~nm}$ with 1200 groove $\mathrm{mm}^{-1}$. The inverse linear dispersion is $5 \mathrm{~nm} \mathrm{~mm}^{-1}$. EA spectra were obtained by focusing the transmitted light onto a photodiode (UDT Sensors) working in photovoltaic mode. The signal was passed through an operational amplifier and measured using a lock-in amplifier (Stanford Instruments SR550) phased with the frequency of the oscillating electric field $(441 \mathrm{~Hz})$ applied to the sample and set to acquire at the second harmonic of the signal. To obtain the EF spectra, luminescence from the sample is collected by an emission monochromator (Acton, 600 groove $\mathrm{mm}^{-1}$ blazed at $500 \mathrm{~nm}$, spectral resolution $5 \mathrm{~nm}$ ) and detected by a water-cooled photomultiplier tube (Hamamatsu R928). The electric signal was pre-amplified before arriving at the lock-in amplifier. The signal was collected at twice the frequency of the oscillating electric field, $75 \mathrm{~Hz}$. The Stark effect on absorption or field-induced change in absorption (EA) at wavenumber $\bar{\nu}, \Delta A(\bar{\nu})$, were fit by a linear combination of zeroth, first, and second derivatives of absorption spectra as follows:

$$
\Delta A(\bar{\nu})=F^{2}\left\{A_{x} A(\bar{\nu})+\frac{B_{x}}{15 h c} \bar{\nu} \frac{\mathrm{d}}{\mathrm{d} \bar{\nu}}\left(\frac{A(\bar{\nu})}{\bar{\nu}}\right)+\frac{C_{x}}{30 h^{2} c^{2}} \bar{\nu} \frac{\mathrm{d}^{2}}{\mathrm{~d} \bar{\nu}^{2}}\left(\frac{A(\bar{\nu})}{\bar{\nu}}\right)\right\}
$$

Similarly, for the field-induced change in fluorescence $(\mathrm{EF})$ :

$$
\Delta I(\bar{\nu})=F^{2}\left\{A_{x}^{\prime} I(\bar{\nu})+\frac{B_{x}^{\prime}}{15 h c} \bar{\nu}^{3} \frac{\mathrm{d}}{\mathrm{d} \bar{\nu}}\left(\frac{I(\bar{\nu})}{\bar{\nu}^{3}}\right)+\frac{C_{x}^{\prime}}{30 h^{2} c^{2}} \bar{\nu}^{3} \frac{\mathrm{d}^{2}}{\mathrm{~d} \bar{\nu}^{2}}\left(\frac{I(\bar{\nu})}{\bar{\nu}^{3}}\right)\right\}
$$

The EA and EF spectra of $\mathrm{Au}_{24}$ are collected in a low-temperature glass at $77 \mathrm{~K}$. The zeroth coefficients $A_{x}$ and $A_{x}^{\prime}$ denote the change in transition moment induced by electric field and the field-induced change in emission intensity, respectively. The first derivative coefficient $B_{x} \cdot\left(B_{x}^{\prime}\right)$ and second derivative coefficient $C_{x} \cdot\left(C_{x}^{\prime}\right)$ denote the spectral shift and spectral broadening of the EA (EF) spectra. These are correlated with the change in polarizability $(\Delta \alpha)$ and dipole moment change $(|\Delta \mu|)$, respectively, in the excited state with respect to the ground state.

High-pressure PL spectroscopy. $\mathrm{Au}_{24}$ in toluene was loaded in a diamond anvil cell high-pressure chamber. Two identical diamond anvils with a culet of $500 \mu \mathrm{m}$ were employed to generate pressure. A stainless-steel gasket was pre-indented to $400 \mu \mathrm{m}$ with a drilled hole $200 \mu \mathrm{m}$ in diameter serving as the sample chamber. The pressure was calibrated using the pressure-dependent ruby fluorescent technique. PL spectra were measured in a Horiba XploRA + Confocal Raman set-up using the $\times 10$ objectives. Samples were excited by the $532 \mathrm{~nm}$ laser and 600 groove $\mathrm{mm}^{-1}$ diffraction grating were adopted.

\section{Data availability}

The source data that support the plots within this paper and other finding of this study are available from the corresponding authors upon request.

Received: 6 January 2020; Accepted: 19 May 2020;

Published online: 09 June 2020

\section{References}

1. Clark, J., Nelson, T., Tretiak, S., Cirmi, G. \& Lanzani, G. Femtosecond torsional relaxation. Nat. Phys. 8, 225-231 (2012).

2. Polli, D. et al. Conical intersection dynamics of the primary photoisomerization event in vision. Nature 467, 440-443 (2010).

3. Chergui, M. \& Collet, E. Photoinduced structural dynamics of molecular systems mapped by time-resolved X-ray methods. Chem. Rev. 117, 11025-11065 (2017).

4. Conyard, J. et al. Ultrafast dynamics in the power stroke of a molecular rotary motor. Nat. Chem. 4, 547-551 (2012).

5. Dereka, B., Koch, M. \& Vauthey, E. Looking at photoinduced charge transfer processes in the IR: answers to several long-standing questions. Acc. Chem. Res. 50, 426-434 (2017).

6. King, A. W., Wang, L. \& Rack, J. J. Excited state dynamics and isomerization in ruthenium sulfoxide complexes. Acc. Chem. Res. 48, 1115-1122 (2015).

7. Iwamura, M., Takeuchi, S. \& Tahara, T. Ultrafast excited-state dynamics of copper(I) complexes. Acc. Chem. Res. 48, 782-791 (2015).

8. Piontkowski, Z. \& McCamant, D. W. Excited-state planarization in donor-bridge dye sensitizers: phenylene versus thiophene bridges. J. Am Chem. Soc. 140, 11046-11057 (2018).

9. Shields, B. J., Kudisch, B., Scholes, G. D. \& Doyle, A. G. Long-lived chargetransfer states of nickel(II) aryl halide complexes facilitate bimolecular photoinduced electron transfer. J. Am. Chem. Soc. 140, 3035-3039 (2018).

10. Sung, J. et al. Direct observation of excimer-mediated intramolecular electron transfer in a cofacially-stacked perylene bisimide pair. J. Am. Chem. Soc. 138, 9029-9032 (2016).

11. Chen, L. X. et al. Capturing a photoexcited molecular structure through timedomain X-ray absorption fine structure. Science 292, 262-264 (2001).

12. Brown-Xu, S. E. et al. Tunable excited-state properties and dynamics as a function of Pt-Pt distance in pyrazolate-bridged Pt(II) dimers. J. Phys. Chem. A 120, 543-550 (2016).

13. Zhou, C. et al. Precise design of phosphorescent molecular butterflies with tunable photoinduced structural change and dual emission. Angew. Chem. Int Ed. 54, 9591-9595 (2015).

14. Druzhinin, S. I. et al. Dynamics of ultrafast intramolecular charge transfer with 4-(dimethylamino)benzonitrile in acetonitrile. J. Phys. Chem. A 110, 2955-2969 (2006).

15. Rettig, W. in Electron Transfer I (ed. Mattay, J.). 253-299 (Springer, Berlin, Heidelberg, 1994).

16. Chi, W. et al. A photoexcitation-induced twisted intramolecular charge shuttle. Angew. Chem. Int. Ed. 58, 7073-7077 (2019).

17. Grabowski, Z. R., Rotkiewicz, K. \& Rettig, W. Structural changes accompanying intramolecular electron transfer: focus on twisted intramolecular charge-transfer states and structures. Chem. Rev. 103, 3899-4032 (2003)

18. Jin, R., Zeng, C., Zhou, M. \& Chen, Y. Atomically precise colloidal metal nanoclusters and nanoparticles: fundamentals and opportunities. Chem. Rev. 116, 10346-10413 (2016).

19. Zhang, H. et al. Bacteria photosensitized by intracellular gold nanoclusters for solar fuel production. Nat. Nanotechnol. 13, 900-905 (2018).

20. Aikens, C. M. Electronic and geometric structure, optical properties, and excited state behavior in atomically precise thiolate-stabilized noble metal nanoclusters. Acc. Chem. Res. 51, 3065-3073 (2018).

21. Pyo, K. et al. Ultrabright luminescence from gold nanoclusters: rigidifying the Au(I)-thiolate shell. J. Am. Chem. Soc. 137, 8244-8250 (2015).

22. Chen, Y. et al. Shortwave infrared in vivo imaging with gold nanoclusters Nano Lett. 17, 6330-6334 (2017).

23. $\mathrm{Du}, \mathrm{B}$. et al. Glomerular barrier behaves as an atomically precise bandpass filter in a sub-nanometre regime. Nat. Nanotechnol. 12, 1096-1102 (2017).

24. Loynachan, C. N. et al. Renal clearable catalytic gold nanoclusters for in vivo disease monitoring. Nat. Nanotechnol. 14, 883-890 (2019).

25. Liu, H. et al. Atomic-precision gold clusters for NIR-II imaging. Adv. Mater. 31, 1901015 (2019).

26. Lei, Z. et al. An atomically precise $\mathrm{Au}_{10} \mathrm{Ag}_{2}$ nanocluster with red-near-IR dual emission. Chem. Eur. J. 22, 11156-11160 (2016)

27. Sugiuchi, M. et al. Aggregation-induced fluorescence-to-phosphorescence switching of molecular gold clusters. J. Am. Chem. Soc. 139, 17731-17734 (2017).

28. Narouz, M. R. et al. Robust, highly luminescent $\mathrm{Au}_{13}$ superatoms protected by N-heterocyclic carbenes. J. Am. Chem. Soc. 141, 14997-15002 (2019).

29. Dass, A., Stevenson, A., Dubay, G. R., Tracy, J. B. \& Murray, R. W. Nanoparticle MALDI-TOF mass spectrometry without fragmentation: $\mathrm{Au}_{25}\left(\mathrm{SCH}_{2} \mathrm{CH}_{2} \mathrm{Ph}\right)_{18}$ and mixed monolayer $\mathrm{Au}_{25}\left(\mathrm{SCH}_{2} \mathrm{CH}_{2} \mathrm{Ph}\right)_{18-\mathrm{x}}(\mathrm{L})_{\mathrm{x}}$. J. Am. Chem. Soc. 130, 5940-5946 (2008).

30. Das, A. et al. Crystal structure and electronic properties of a thiolate-protected $\mathrm{Au}_{24}$ nanocluster. Nanoscale 6, 6458-6462 (2014). 
31. Yang, S. et al. Total structure determination of $\mathrm{Au}_{16}(\mathrm{~S}-\mathrm{Adm})_{12}$ and $\mathrm{Cd}_{1} \mathrm{Au}_{14}(\mathrm{StBu})_{12}$ and implications for the structure of $\mathrm{Au}_{15}(\mathrm{SR})_{13} . \mathrm{J} . \mathrm{Am}$. Chem. Soc. 140, 10988-10994 (2018).

32. Pei, Y. et al. Interlocked catenane-like structure predicted in $\mathrm{Au}_{24}(\mathrm{SR})_{20}$ : implication to structural evolution of thiolated gold clusters from homoleptic gold(I) thiolates to core-stacked nanoparticles. J. Am. Chem. Soc. 134, 3015-3024 (2012).

33. Vieira dos Santos, F. J. \& de Castro, C. A. N. Viscosity of toluene and benzene under high pressur. Int. J. Thermophys. 18, 367-378 (1997).

34. Mehata, M. S. Enhancement of charge transfer and quenching of photoluminescence of capped CdS quantum dots. Sci. Rep. 5, 1-11 (2015).

35. Moscatelli, A. et al. Electric-field-induced fluorescence quenching in polyfluorene, ladder-type polymers, and MEH-PPV: evidence for field effects on internal conversion rates in the low concentration limit. J. Phys. Chem. B 114, 14430-14439 (2010).

36. Oh, D. H., Sano, M. \& Boxer, S. G. Electroabsorption (Stark effect) spectroscopy of mono- and biruthenium charge-transfer complexes: measurements of changes in dipole moments and other electrooptic properties. J. Am. Chem. Soc. 113, 6880-6890 (1991).

37. Kodali, G., Siddiqui, S. U. \& Stanley, R. J. Charge redistribution in oxidized and semiquinone E. coli DNA photolyase upon photoexcitation: Stark spectroscopy reveals a rationale for the position of Trp382. J. Am. Chem. Soc. 131, 4795-4807 (2009).

38. Song, Y. et al. Crystal structure of selenolate-protected $\mathrm{Au}_{24}(\mathrm{SeR})_{20}$ nanocluster. J. Am. Chem. Soc. 136, 2963-2965 (2014).

39. Li, Q. et al. A mono-cuboctahedral series of gold nanoclusters: photoluminescence origin, large enhancement, wide tunability, and structure-property correlation. J. Am. Chem. Soc. 141, 5314-5325 (2019).

40. Zhou, M. et al. Evolution of excited-state dynamics in periodic $\mathrm{Au}_{28}, \mathrm{Au}_{36}$, $\mathrm{Au}_{44}$, and $\mathrm{Au}_{52}$ nanoclusters. J. Phys. Chem. Lett. 8, 4023-4030 (2017).

41. Yam, V. W.-W., Au, V. K.-M. \& Leung, S. Y.-L. Light-emitting self-assembled materials based on $\mathrm{d} 8$ and $\mathrm{d} 10$ transition metal complexes. Chem. Rev. 115, 7589-7728 (2015).

42. Huang, R.-W. et al. Hypersensitive dual-function luminescence switching of a silver-chalcogenolate cluster-based metal-organic framework. Nat. Chem. 9, 689-697 (2017).

43. Locknar, S. A., Chowdhury, A. \& Peteanu, L. A. Matrix and temperature effects on the electronic properties of conjugated molecules: an electroabsorption study of all-trans-retinal. J. Phys. Chem. B 104, 5816-5824 (2000).

44. Smith, T. M., Hazelton, N., Peteanu, L. A. \& Wildeman, J. Electrofluorescence of MEH-PPV and its oligomers: evidence for field-induced fluorescence quenching of single chains. J. Phys. Chem. B 110, 7732-7742 (2006).

\section{Acknowledgements}

This work was financially supported by the National Science Foundation (DMR-1808675 and DMR- 2002936/2002891). We thank Ms. Jingchun Huang for the preliminary
PL measurements and Dr. Meng Zhou for the preliminary TA measurements. This research used resources of the Center for Functional Nanomaterials, which is a U.S DOE Office of Science Facility at Brookhaven National Laboratory under Contract No. DE-SC0012704.

\section{Author contributions}

Q.L. conceived the project and designed the research advised by R.J. and X.W.G. Q.L. and D.Z. performed the steady-state and TCSPC experiments with help from H.Z., T.C., O.C., M.C., and M.L. D.Z. and H.Z. performed the fs-TA experiments and analyzed the fs-TA data. W.Y.S. and L.A.P. conducted the Stark measurements. Q.L. and X.W.G. conducted high-pressure measurements. Q.L. and J.C. synthesized the samples. Q.L., D.Z., H.Z., R.J. and X.W.G wrote the paper, and all authors commented on it.

\section{Competing interests}

The authors declare no competing interests.

\section{Additional information}

Supplementary information is available for this paper at https://doi.org/10.1038/s41467020-16686-8.

Correspondence and requests for materials should be addressed to X.W.G., H.Z. or R.J

Peer review information Nature Communications thanks the anonymous reviewers for their contribution to the peer review of this work. Peer reviewer reports are available.

Reprints and permission information is available at http://www.nature.com/reprints

Publisher's note Springer Nature remains neutral with regard to jurisdictional claims in published maps and institutional affiliations.

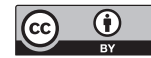

Open Access This article is licensed under a Creative Commons Attribution 4.0 International License, which permits use, sharing, adaptation, distribution and reproduction in any medium or format, as long as you give appropriate credit to the original author(s) and the source, provide a link to the Creative Commons license, and indicate if changes were made. The images or other third party material in this article are included in the article's Creative Commons license, unless indicated otherwise in a credit line to the material. If material is not included in the article's Creative Commons license and your intended use is not permitted by statutory regulation or exceeds the permitted use, you will need to obtain permission directly from the copyright holder. To view a copy of this license, visit http://creativecommons.org/ licenses/by/4.0/

(c) The Author(s) 2020 